\title{
El istmo centroamericano. Trazas en la historia
}

\author{
Central American Isthmus. Traces in history \\ Luis RodríGuez CASTILLO* \\ do) 10.29043 /liminar.v20i1.900
}

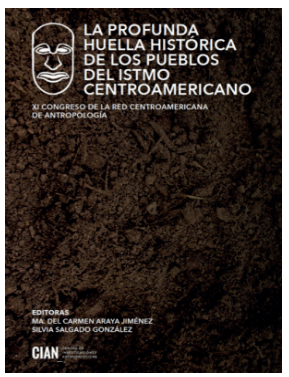

Resumen: Reseña de La profunda huella histórica de los pueblos del istmo centroamericano: XI Congreso de la Red Centroamericana de Antropología, editado por María del Carmen Araya Jiménez y Silvia Salgado González. San José, Costa Rica: Centro de Investigaciones Antropológicas-Universidad de Costa Rica, 2020. http://www.kerwa.ucr.ac.cr/handle/10669/82758

Abstract: Review of La profunda huella histórica de los pueblos del istmo centroamericano: XI Congreso de la Red Centroamericana de Antropología, editado por María del Carmen Araya Jiménez y Silvia Salgado González. San José, Costa Rica: Centro de Investigaciones Antropológicas-Universidad de Costa Rica, 2020. http://www.kerwa.ucr.ac.cr/handle/10669/82758

\section{Un largo, pero necesario, introito}

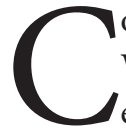

omo una forma espectacular de iniciar su estudio intitulado Europa y la gente sin Historia, Eric R. Wolf, afirmaba que "la antropología necesitaba descubrir a la historia, a una historia que pudiera explicar las formas en que el sistema social del mundo moderno llegó a ser lo que es, y que se esforzara por atribuir un sentido analítico a todas las sociedades" (Wolf, 1987, p. 9). Sin pretender ser iconoclasta, Wolf estaba en un error. A la antropología no le faltaba ese descubrimiento, ya lo tenía. A México, San Cristóbal de Las Casas, Chiapas, México 
contrasentido, y reverenciando a nuestros padres fundadores, la antropología ha sido la madre de todas las ciencias sociales y las humanidades. ${ }^{1}$

Afirmo que esta es una vieja relación porque, en la evolución del pensamiento antropológico, en el momento en que se desprenden las ciencias de la filosofía se definió la antropología, afirma Marvin Harris, como la ciencia de la evolución y la historia humana (Harris, 1996). No obstante, en lo que toca a la legitimación que llevará a la institucionalización de la antropología social, tanto Alfred Reginal Radcliffe-Brown (1975) como Sir Edward Evan Evans-Pritchard (1990) argumentaron a favor de la existencia de dos "métodos diferentes" para explicar los hechos culturales que deberían mantenerse cuidadosamente separados: por un lado, uno especulativo-filosófico sobre lo que fueron y podrían ser las sociedades humanas y, por el otro, uno positivo que aspire a descubrir leyes generales de los fenómenos culturales que deberían seguir los métodos lógicos ordinarios de las ciencias naturales.

En ese sentido, Evans-Pritchard afirmó que "para la comprensión del funcionamiento de una sociedad, el investigador no necesita conocer su historia, de igual modo que un fisiólogo no necesita conocer la historia de un organismo para entenderlo. Ambos son sistemas naturales y pueden ser descritos en términos de leyes naturales sin recurrir a la historia" (Evans-Pritchard, 1990, p. 11). Pero recordemos que esto no implica a la antropología en su totalidad como tradición, sino solo a la rama de la antropología social y en un momento particular: el de su institucionalización. Siguiendo el modelo de Thomas Samuel Kuhn (2004), se requerían fuentes de legitimidad que los padres fundadores tenían en el paradigma seguido por las ciencias naturales y su búsqueda de leyes generales. Por su parte, Eric R. Wolf, con su espectacular arranque a Europa y la gente sin historia, marca los puntos de enfrentamiento, con el paradigma hegemónico, de su contexto y momento histórico.

Sin embargo, como lo demuestra el libro La profunda huella..., las ciencias antropológicas centroamericanas no necesitan buscar esa historia. Esta relación es un campo de añoso trillado en nuestra región, pues habrá que recordar que el polaco Bronislaw Malinowski, en un breve ensayo publicado originalmente en castellano en Cuba en 1940 como introducción a la obra Contrapunteo cubano del tabaco y el azúcar, de Fernando Ortiz, ${ }^{2}$ elogió esa relación:

El presente libro es una obra maestra de investigación histórica y sociológica, tan magistralmente condensada y documentada como libre de toda erudición pedante y estéril [sus argumentos] podrían ser usados como cartas de rumbo para emprender respectivos trabajos de investigación en el campo de la etnografía (Malinowski, 1983, p. ii).

Dicha relación ha sido tan estrecha en la antropología mundial que el mismo Evans-Pritchard, en su disertación en The Marett Lecture el 3 de junio de 1950 en Oxford, afirmó que la antropología estaba más cerca de la historia y del conjunto de las humanidades que de las ciencias explicativas (ciencias duras).

\footnotetext{
${ }^{1}$ Una versión preliminar de esta colaboración fue parte de la presentación del libro realizada en modalidad virtual el viernes 25 de septiembre de 2020, organizada por el Centro de Investigaciones Antropológicas (CIAN) de la Universidad de Costa Rica (UCR).

${ }^{2}$ Se reconoce, por cierto, que en este libro se introdujo el concepto de "transculturación" en el debate de las ciencias sociales y antropológicas en América Latina (Martí, 2011). En el libro que aquí comento dos colaboradores retoman dos perspectivas semejantes utilizadas por Ortiz: Ibarra (pp. 59-80) para el análisis desde un producto para desentrañar el conjunto de relaciones sociales y Bartolomé (pp. 98-117) en cuanto a las relaciones entre diferentes culturas, llamadas por este "interétnicas".
} 
La tesis que planteó fue que la antropología es un tipo de historiografía porque:

implica que estudia a las sociedades como si fueran sistemas éticos y no sistemas naturales, que está interesada en el diseño antes que en el proceso y, por eso, busca patrones y no leyes científicas [generales], interpretaciones y no explicaciones [... así] la desprendemos de los dogmas filosóficos esenciales y le damos la oportunidad así de ser realmente empírica, por paradójico que pueda parecer, y científica en el verdadero sentido de la palabra (Evans-Pritchard, 1990, pp. 19-20).

La referencia al trabajo de Wolf que, desde mi punto de vista, es pionero en lo que más tarde se denominó como antropología de la globalización, no es gratuita, pues más de algún despistado podría pensar que el libro La profunda huella histórica de los pueblos del istmo centroamericano, por su título, o es un libro de historia de Centroamérica o es un libro de antropología que quiere seguir la sugerencia de Wolf. Pues bien, ninguna de las dos.

Y, por ello, enseguida abordo brevemente los contenidos de los capítulos, sigo con destacar las aportaciones de este libro, y a manera de cierre expongo lo que para mí es la oportunidad de esta obra como un exhorto a su lectura.

\section{Contenidos}

La profunda huella histórica de los pueblos del istmo centroamericano es un libro que condensa, en cinco capítulos, profundos conocimientos antropológicos sobre el istmo centroamericano que, sin hacer una discusión explícita de esto, retoman la perspectiva del análisis holístico de la tradición adjudicada a Boas. Es decir, las distintas aportaciones concentran en una misma reflexión los descubrimientos realizados desde la lingüística, la arqueología, la antropología física o biológica y la etnología o antropología social en torno a un tópico de investigación. Esto es así porque reúne las ponencias magistrales del XI Congreso de la Red Centroamericana de Antropología (RCA) cuyos autores, de una reconocida trayectoria y aportes, discuten, en mayor o menor grado, las contribuciones que hicieron al conocimiento de la región los antropólogos a quienes se les hizo un reconocimiento en dicho congreso. Recordemos que fue dedicado al Dr. Richard Cooke, al Dr. Ramiro Barrantes Mesén, al Dr. Adolfo Constela Umaña y al Mtro. Oscar Fonseca Zamora. Aunque no están exentas sus disertaciones del diálogo con ellos desde una síntesis de sus propias reflexiones acumuladas sobre el tiempo.

Así, Marcos Guevara Berger nos presenta en el trabajo "Istmicidad. Persistencia de la historia y estrechos dudosos" (pp.16-35) la existencia de una doble interpretación de esa idea con diferente raigambre histórica: por un lado la precolonial como una continuidad de relaciones y, por otro, que proviene con la historia de la conquista como un estrecho que separa (mares y continentes), que fue construido a partir de procesos históricos, geográficos y productivos; pero sobre todo es un estrecho dudoso, ya que ha sido influido por razones geopolíticas y por los procesos de la construcción de los Estados-nación centroamericanos. A través de los aportes de los homenajeados en esa edición del congreso se recupera información histórica y arqueológica para constatar su argumento de una continuidad ístmica.

Guevara retoma desde los postulados de Cook, Constela y Fonseca, además de la historia, el ejercicio metodológico comparativo para dilucidar diferentes formas de istmicidad: desde la lingüística, desde la arqueología y desde la comparación iconográfica presentes en códices, así como en la recuperación 
y comparación de mitos. E, incluso, pasa revista a lo hecho por la antropología y sus congresos para favorecer, o no, la construcción de la istmicidad.

Gloria Lara Pinto en "Geopolítica de la identidad étnica y lingüística del istmo centroamericano. Una propuesta desde el territorio ancestral lenca” (pp. 36-58) se propone, desde una perspectiva lingüística e histórica, renovar la interpretación de la historia antigua del istmo centroamericano, para lo cual afirma, siguiendo a Bloch, "la pauta a seguir — en apariencia sencilla- consiste en esbozar el marco comparativo adecuado para reconocer los fenómenos generales, pero sin subestimar el poder explicativo de las condiciones locales" (p. 38). Así, argumenta que la resiliencia del pueblo lenca solo puede explicarse en la historia de largo aliento, y valida la tesis de Constenla sobre la existencia del "microfilo lenmichí" (lenca, misumalpa y chibcha) en Centroamérica.

De esta manera, la autora propone que debemos pensar Centroamérica fuera del paradigma que encasilla la región en dos áreas culturales, para pensar en formaciones culturales de múltiples zonas y fronteras y, por ende, de interinfluencias: la zona norte, la zona sur y la zona central, esta de su interés y que conforma grosso modo los actuales países de Honduras, Nicaragua y El Salvador. Con esta mirada de largo aliento puede dar cuenta de que la imagen de un territorio vacío es un mito y de la presencia del grupo lenca al menos 10000 años antes del presente.

"Producción y redes de intercambio en el sur de América Central en el siglo XVI. Una perspectiva desde el estudio de la sal" (pp. 59-80), de Eugenia Ibarra Rojas, retoma resultados de la arqueología, la lingüística y la etnohistoria propuestos por los homenajeados, y los ubica en el marco más general del modelo del "control vertical de un máximo de pisos ecológicos" para reconstruir el panorama de la producción y circulación de la sal desde tiempos prehispánicos en el territorio de la actual Centroamérica.

Retoma que estos autores documentaron cómo los cacicazgos lucharon por obtener y controlar áreas en diferentes pisos y descubrieron el patrón en torno a tres rasgos: recursos escasos, como la sal y el oro, cercanía de rutas de navegación a corta y a larga distancia y conexiones con tramos terrestres que facilitaron la protección de los sitios, así como la entrada y salida de bienes. También que la sal fue un producto importante como parte de los tributos, tanto prehispánico como colonial. Finalmente, observa que la explotación y distribución de la sal durante la Colonia estuvieron ligadas a la ganadería.

Por su parte, Ernesto Vargas Pacheco, en su contribución intitulada "El occidente de México y los posibles contactos con Centro y Sudamérica. La ruta del Pacífico" (pp. 81-97), expone su análisis a través de las evidencias arqueológicas como cerámicas tempranas, las tumbas de tiro, la metalurgia, la arquitectura y los petroglifos como sustento de la tesis de los contactos tempranos entre estas regiones, principalmente de relaciones comerciales a través del océano Pacífico. Una de las hipótesis que presenta es la expansión de la ruta desde el sur en la búsqueda de las conchas de spondylus - que llegaron a nombrarse "el oro rojo inca"-.

A la par de presentar la datación de los complejos culturales a lo largo del Pacífico, hace un análisis comparativo de las evidencias de cultura material antes señaladas entre Centroamérica, Sudamérica y el occidente de México, integrada esta última región por los actuales estados de Sinaloa, Nayarit, Colima, Jalisco, Michoacán y Guerrero.

Cierra es libro el capítulo "Los rostros étnicos de México. Relaciones interétnicas, identidades y autonomías" (pp. 98-117), de Miguel Alberto Bartolomé Bistoletti, en el que el autor pasa revista a la política indigenista en México que, de haber tenido éxito, hubiera generado, según su punto de vista, una "configuración social empobrecida e híbrida" (p. 103), pues es una política pública que no tomó en 
consideración la opinión de su población objetivo: los pueblos indígenas. Estos pueblos han sido materia de discriminación y racismo, de modo que en el caso de México se cumple la máxima: "tenemos el prejuicio de no tener prejuicios". Es decir, adolecemos de una sistemática negación de nuestro racismo y hasta de nuestra propia mestizofilia.

Destaca, desde luego, la importancia del Ejercito Zapatista de Liberación Nacional para dar mayor visibilidad a los movimientos étnicos y a los procesos de etnogénesis en México, y que las reconstituciones identitarias, como dato objetivo de la realidad, son también una construcción simbólica, signos que nos permiten comunicarnos en nuestros sistemas culturales.

\section{Aportaciones}

En el conjunto observo los siguientes aspectos en los que los capítulos del libro hacen aportes y que serán de gran riqueza en los procesos de formación antropológica en la región una vez que se ha publicado este libro en un formato de libre acceso en la página http://www.kerwa.ucr.ac.cr/handle/10669/82758.

1. El conocimiento, uso adecuado e interpelación de modelos establecidos tal como lo hacen los autores y autoras en sus correspondientes capítulos, principalmente en los primeros tres, de Guevara, Lara e Ibarra.

2. Se llama a la imposibilidad de la idea de aislamiento de los procesos sociales a través del énfasis en el intercambio de bienes materiales en dos capítulos (Ibarra y Vargas); uno da cuenta de las líneas de continuidad y discontinuidad histórica y geográfica (Ibarra) y, el otro, (Vargas) destaca las tesis planteadas por Guevara en relación con el istmo como puente y punto de partida para los intercambios interregionales. Ambos muestran la existencia de complejas y eficientes redes de intercambio en el istmo: sal y ganado en el análisis de Ibarra, y múltiples objetos materiales comparables entre el occidente de México y las culturas andinas de Ecuador y Perú que mantienen una identidad estilística y simbólica en lo que Vargas denomina "ruta del Pacífico".

3. Señalan que los intercambios materiales no son aislados, les acompañan los intercambios de carácter cultural. Esto es patente en la totalidad de los autores del libro, pero en particular Bartolomé remarca, en el caso de significados e intercambios regionales, los intercambios de ideas en las prácticas político-culturales de la etnicidad que, aunque enfocados en un caso nacional, muestran la necesidad de comprenderlos en el ámbito de las relaciones regionales.

4. La previsión epistemológica presente en todos los capítulos en relación con los problemas que se nos presentan con la sobregeneralización y homogeneización que se exteriorizan en el uso acrítico de ciertas categorías, incluidas las propuestas por los autores (istmicidad, zona intermedia, Mesoamérica, indígena, etcétera).

5. La sugerencia de cuestionar las historiografías de la región desde una mirada colonialista, es decir, la que arranca desde la llegada de los españoles y, por el contrario, de atender los procesos sociales de "larga duración" (especialmente en la contribución de Lara).

6. Aunado a lo anterior, los autores aportan en el sentido de que los científicos sociales no debemos olvidar que gran parte de nuestra historia regional y de las historias nacionales devienen de intereses geopolíticos de los Estados por encontrar, primero, y controlar, después, un paso transoceánico. 
7. La discusión, abierta y apasionante, sobre las fronteras, en torno a las que exponen acuerdos y desacuerdos sobre el uso de esta noción.

8. Considero, entonces, que desde sus posiciones y tópicos de investigación central cada uno de los autores y autoras de los capítulos construye un "estado de la cuestión” y presenta las claves para avanzar en la investigación antropológica contemporánea sobre la región.

Aún con todas estas virtudes, cierto es que no existe libro o capítulo perfecto. Así, Guevara presenta interesantes reflexiones sobre las fronteras cambiantes, pero no su propia definición de dicha categoría. Y la relación con la configuración de los Estados pareciera una visión reduccionista a la de "límite". Lara Pinto usa un concepto muy moderno, como lo es "geopolítica", que según Nogué y Rufí (2001) solo es susceptible de aplicarse a los Estados-nación, en tanto que, coincidiendo con María Eugenia Bozzoli (2006) en torno a que los cacicazgos, como jefaturas complejas y con funciones diferenciadas, solo se aproximaban a una sociedad estatal que puede relacionarse, a su vez, con otras semejantes, formando federaciones y confederaciones. Por su parte, a Ibarra considero que le falta una discusión más amplia sobre el modelo y los pisos ecológicos, y al final repite con una mirada poco crítica que hay un uso y control de los pisos, pero tampoco aclara cómo el desarrollo de la ganadería que se dio en la zona de la región histórica chibcha, y desde luego cómo el incremento de la demanda cambió la producción y las rutas de la sal.

Vargas, si bien presenta un panorama ecuánime, en su conclusión vuelve a dejar sembrada la duda cuando afirma que: "Los indicios para hablar de contactos se deben fundamentalmente a que existen similitudes en ciertos artefactos, cerámicas, figurillas, arquitectura y en el uso de ciertas tecnologías, lo que no necesariamente indica relaciones" (p. 97). De igual forma, Bartolomé estructura un discurso que al llegar a la conclusión deja la impresión de que reifica lo que había criticado, que la identidad étnica es una construcción ideológica.

Esta es una lectura personal y, desde luego, la mejor opinión se la podrán formar los lectores. Por ello, comento enseguida por qué debe ser leída.

\section{Oportunidad y exhorto a la lectura}

La publicación de este libro es oportuna porque no contamos con un texto semejante que ofrezca una discusión actualizada en relación con las investigaciones sobre la región y las definiciones a retomar en nuestros procesos investigativos en curso y en el desarrollo de la investigación sobre la "istmicidad" contemporánea.

El libro es pertinente ya que destaca en cada uno de los capítulos una serie de discusiones desde las diferentes subdisciplinas antropológicas para definir, desde una mirada contemporánea, la región. Esto sin olvidar los procesos sociohistóricos ni las evidencias arqueológicas que conforman la región centroamericana y, particularmente, sus implicaciones como región ístmica y puente de comunicación. Discuten claramente sin rechazar categorías que llaman a la generalización, pero también homogeneizan la región desde diversas categorías establecidas en la discusión antropológica: frontera, área cultural, istmo centroamericano, zona intermedia, Mesoamérica, entre otras.

La obra representa, desde luego, una gran oportunidad en torno a la proyección social del Centro de Investigaciones Antropológicas (CIAN) de la Universidad de Costa Rica (UCR), como un centro de producción de conocimiento científico en Costa Rica que contribuirá al fortalecimiento de una antropo- 
logía con identidad ístmica y centroamericana y dará cuenta de la historia profunda de la antropología en nuestra macrorregión mesoamericana.

Por eso es necesario leer este libro que generosamente han coordinado María del Carmen Araya Jiménez y Silvia Salgado González, para que podamos gozar en reconocer la profunda huella de nuestra istmicidad y así comprendernos en nuestro espacio centroamericano y de frontera.

\section{Referencias}

Bozzoli, M. E. (2006). Oí decir del usékar. Editorial Universidad Estatal a Distancia.

Evans-Pritchard, E. E. (1990). Ensayos de antropología social. Siglo XXI.

Harris, M. (1996). El desarrollo de las teorías antropológicas. Historia de las teorías de la cultura. Siglo XXI.

Kuhn, T. S. (2004). La estructura de las revoluciones científicas (Colección Breviarios). Fondo de Cultura Económica.

Malinowski, B. (1983). Introducción. En F. Ortiz, Contrapunteo cubano el tabaco y el azúcar (Advertencia de sus contrastes agrarios, económicos, históricos y sociales, su etnografía y su transculturación) (Colección Pensamiento Cubano). Editorial de Ciencias Sociales (original publicado en 1940).

Martí, A. J. (2011). Contrapunteo etnológico: el debate aculturación o transculturación desde Fernando Ortiz hasta nuestros días. Kálathos. Revista Transdisciplinaria Metro-Inter, 4(2).

Nogué, J., y Rufí, V. (2001). Geopolítica, identidad y globalización (Colección Ariel Geografía). Ariel.

Radcliffe-Brown, A. R. (1975). El método de la antropología social (Colección Antropología). Anagrama.

Wolf, E.R. (1987). Europa y la gente sin historia. Fondo de Cultura Económica. 